\title{
Ultrahigh speed endoscopic optical coherence tomography using micromotor imaging catheter and VCSEL technology
}

\author{
Tsung-Han Tsai, ${ }^{1}$ Benjamin Potsaid, ${ }^{1,2}$ Yuankai K. Tao, ${ }^{1}$ Vijaysekhar Jayaraman, ${ }^{3}$ \\ James Jiang, ${ }^{2}$ Peter J. S. Heim, ${ }^{4}$ Martin F. Kraus, ${ }^{1,5}$ Chao Zhou, ${ }^{1}$ Joachim Hornegger, ${ }^{5}$ \\ Hiroshi Mashimo, ${ }^{6}$ Alex E. Cable, ${ }^{2}$ and James G. Fujimoto ${ }^{1, *}$ \\ ${ }^{1}$ Department of Electrical Engineering and Computer Science and Research Laboratory of Electronics, \\ Massachusetts Institute of Technology, Cambridge, MA 02139, USA \\ ${ }^{2}$ Advanced Imaging Group, Thorlabs, Inc., Newton, NJ, USA \\ ${ }^{3}$ Praevium Research, Inc., Santa Barbara, CA, USA \\ ${ }^{4}$ Thorlabs Quantum Electronics, Inc., Jessup, MD, USA \\ ${ }^{5}$ Pattern Recognition Lab and Graduate School in Advanced Optical Technologies, \\ University Erlangen-Nuremberg, Germany \\ ${ }^{6}$ Veterans Affairs Healthcare System Boston and Harvard Medical School, Boston, MA, USA \\ *jgfuji@mit.edu
}

\begin{abstract}
We developed a micromotor based miniature catheter with an outer diameter of $3.2 \mathrm{~mm}$ for ultrahigh speed endoscopic swept source optical coherence tomography (OCT) using a vertical cavity surfaceemitting laser (VCSEL) at a $1 \mathrm{MHz}$ axial scan rate. The micromotor can rotate a micro-prism at several hundred frames per second with less than 5 $\mathrm{V}$ drive voltage to provide fast and stable scanning, which is not sensitive to the bending of the catheter. The side-viewing probe can be pulled back to acquire a three-dimensional (3D) data set covering a large area on the specimen. The VCSEL provides a high axial scan rate to support dense sampling under high frame rate operation. Using a high speed data acquisition system, in vivo 3D-OCT imaging in the rabbit GI tract and ex vivo imaging of a human colon specimen with $8 \mu \mathrm{m}$ axial resolution, $8 \mu \mathrm{m}$ lateral resolution and $1.2 \mathrm{~mm}$ depth range in tissue at a frame rate of $400 \mathrm{fps}$ was demonstrated.
\end{abstract}

(C)2013 Optical Society of America

OCIS codes: (170.4500) Optical coherence tomography; (170.3880) Medical and biological imaging; (170.2150) Endoscopic imaging; (170.2680) Gastrointestinal; (140.3600) Threedimensional image acquisition; (110.2350) Fiber optics imaging; (120.5800) Scanners; (120.3890) Medical optics instrumentation.

\section{References and links}

1. D. Huang, E. A. Swanson, C. P. Lin, J. S. Schuman, W. G. Stinson, W. Chang, M. R. Hee, T. Flotte, K. Gregory, C. A. Puliafito, and J. G. Fujimoto, "Optical coherence tomography," Science 254(5035), 1178-1181 (1991).

2. G. J. Tearney, S. A. Boppart, B. E. Bouma, M. E. Brezinski, N. J. Weissman, J. F. Southern, and J. G. Fujimoto, "Scanning single-mode fiber optic catheter-endoscope for optical coherence tomography," Opt. Lett. 21(7), 543545 (1996).

3. G. J. Tearney, M. E. Brezinski, B. E. Bouma, S. A. Boppart, C. Pitris, J. F. Southern, and J. G. Fujimoto, "In vivo endoscopic optical biopsy with optical coherence tomography," Science 276(5321), 2037-2039 (1997).

4. S. H. Yun, G. J. Tearney, B. J. Vakoc, M. Shishkov, W. Y. Oh, A. E. Desjardins, M. J. Suter, R. C. Chan, J. A. Evans, I.-K. Jang, N. S. Nishioka, J. F. de Boer, and B. E. Bouma, "Comprehensive volumetric optical microscopy in vivo," Nat. Med. 12(12), 1429-1433 (2007).

5. D. C. Adler, Y. Chen, R. Huber, J. Schmitt, J. Connolly, and J. G. Fujimoto, "Three-dimensional endomicroscopy using optical coherence tomography," Nat. Photonics 1(12), 709-716 (2007).

6. B. J. Vakoc, M. Shishko, S. H. Yun, W. Y. Oh, M. J. Suter, A. E. Desjardins, J. A. Evans, N. S. Nishioka, G. J. Tearney, and B. E. Bouma, "Comprehensive esophageal microscopy by using optical frequency-domain imaging (with video)," Gastrointest. Endosc. 65(6), 898-905 (2007). 
7. H. L. Fu, Y. X. Leng, M. J. Cobb, K. Hsu, J. H. Hwang, and X. D. Li, "Flexible miniature compound lens design for high-resolution optical coherence tomography balloon imaging catheter," J. Biomed. Opt. 13(6), 060502 (2008).

8. M. J. Suter, B. J. Vakoc, P. S. Yachimski, M. Shishkov, G. Y. Lauwers, M. Mino-Kenudson, B. E. Bouma, N. S. Nishioka, and G. J. Tearney, "Comprehensive microscopy of the esophagus in human patients with optical frequency domain imaging," Gastrointest. Endosc. 68(4), 745-753 (2008).

9. M. J. Suter, P. A. Jillella, B. J. Vakoc, E. F. Halpern, M. Mino-Kenudson, G. Y. Lauwers, B. E. Bouma, N. S. Nishioka, and G. J. Tearney, "Image-guided biopsy in the esophagus through comprehensive optical frequency domain imaging and laser marking: a study in living swine," Gastrointest. Endosc. 71(2), 346-353 (2010).

10. J. F. Xi, L. Huo, Y. C. Wu, M. J. Cobb, J. H. Hwang, and X. D. Li, "High-resolution OCT balloon imaging catheter with astigmatism correction," Opt. Lett. 34(13), 1943-1945 (2009).

11. W. Kang, H. Wang, Y. S. Pan, M. W. Jenkins, G. A. Isenberg, A. Chak, M. Atkinson, D. Agrawal, Z. L. Hu, and A. M. Rollins, "Endoscopically guided spectral-domain OCT with double-balloon catheters," Opt. Express 18(16), 17364-17372 (2010).

12. A. Sergeev, V. Gelikonov, G. Gelikonov, F. Feldchtein, R. Kuranov, N. Gladkova, N. Shakhova, L. Snopova, A. Shakhov, I. Kuznetzova, A. Denisenko, V. Pochinko, Y. Chumakov, and O. Streltzova, "In vivo endoscopic OCT imaging of precancer and cancer states of human mucosa," Opt. Express 1(13), $432-440$ (1997).

13. X. M. Liu, M. J. Cobb, Y. C. Chen, M. B. Kimmey, and X. D. Li, "Rapid-scanning forward-imaging miniature endoscope for real-time optical coherence tomography," Opt. Lett. 29(15), 1763-1765 (2004).

14. A. D. Aguirre, J. Sawinski, S. W. Huang, C. Zhou, W. Denk, and J. G. Fujimoto, "High speed optical coherence microscopy with autofocus adjustment and a miniaturized endoscopic imaging probe," Opt. Express 18(5), 42224239 (2010).

15. T.-H. Tsai, B. Potsaid, M. F. Kraus, C. Zhou, Y. K. Tao, J. Hornegger, and J. G. Fujimoto, "Piezoelectrictransducer-based miniature catheter for ultrahigh-speed endoscopic optical coherence tomography," Biomed. Opt. Express 2(8), 2438-2448 (2011).

16. Y. T. Pan, H. K. Xie, and G. K. Fedder, "Endoscopic optical coherence tomography based on a microelectromechanical mirror," Opt. Lett. 26(24), 1966-1968 (2001).

17. W. Jung, D. T. McCormick, J. Zhang, L. Wang, N. C. Tien, and Z. P. Chen, “Three-dimensional endoscopic optical coherence tomography by use of a two-axis microelectromechanical scanning mirror," Appl. Phys. Lett. 88(16), 163901 (2006).

18. K. H. Kim, B. H. Park, G. N. Maguluri, T. W. Lee, F. J. Rogomentich, M. G. Bancu, B. E. Bouma, J. F. de Boer, and J. J. Bernstein, "Two-axis magnetically-driven MEMS scanning catheter for endoscopic high-speed optical coherence tomography," Opt. Express 15(26), 18130-18140 (2007).

19. J. J. Sun, S. G. Guo, L. Wu, L. Liu, S. W. Choe, B. S. Sorg, and H. K. Xie, "3D in vivo optical coherence tomography based on a low-voltage, large-scan-range 2D MEMS mirror,” Opt. Express 18(12), 12065-12075 (2010).

20. P. H. Tran, D. S. Mukai, M. Brenner, and Z. Chen, "In vivo endoscopic optical coherence tomography by use of a rotational microelectromechanical system probe," Opt. Lett. 29(11), 1236-1238 (2004).

21. P. R. Herz, Y. Chen, A. D. Aguirre, K. Schneider, P. Hsiung, J. G. Fujimoto, K. Madden, J. Schmitt, J. Goodnow, and C. Petersen, "Micromotor endoscope catheter for in vivo, ultrahigh-resolution optical coherence tomography," Opt. Lett. 29(19), 2261-2263 (2004).

22. S.-W. Lee, A. E. Heidari, D. Yoon, D. Mukai, T. Ramalingam, S. Mahon, J. Yin, J. Jing, G. Liu, Z. Chen, and M. Brenner, "Quantification of airway thickness changes in smoke-inhalation injury using in-vivo 3-D endoscopic frequency-domain optical coherence tomography," Biomed. Opt. Express 2(2), 243-254 (2011).

23. S. Liang, A. Saidi, J. Jing, G. Liu, J. Li, J. Zhang, C. Sun, J. Narula, and Z. Chen, "Intravascular atherosclerotic imaging with combined fluorescence and optical coherence tomography probe based on a double-clad fiber combiner," J. Biomed. Opt. 17(7), 070501 (2012).

24. J. Li, M. de Groot, F. Helderman, J. Mo, J. M. A. Daniels, K. Grünberg, T. G. Sutedja, and J. F. de Boer, "High speed miniature motorized endoscopic probe for optical frequency domain imaging," Opt. Express 20(22), $24132-24138$ (2012).

25. S. R. Chinn, E. A. Swanson, and J. G. Fujimoto, "Optical coherence tomography using a frequency-tunable optical source," Opt. Lett. 22(5), 340-342 (1997).

26. B. Golubovic, B. E. Bouma, G. J. Tearney, and J. G. Fujimoto, "Optical frequency-domain reflectometry using rapid wavelength tuning of a $\mathrm{Cr}^{4+}$ :forsterite laser," Opt. Lett. 22(22), 1704-1706 (1997).

27. M. A. Choma, M. V. Sarunic, C. H. Yang, and J. A. Izatt, "Sensitivity advantage of swept source and Fourier domain optical coherence tomography," Opt. Express 11(18), 2183-2189 (2003).

28. S. H. Yun, C. Boudoux, G. J. Tearney, and B. E. Bouma, "High-speed wavelength-swept semiconductor laser with a polygon-scanner-based wavelength filter," Opt. Lett. 28(20), 1981-1983 (2003).

29. S. H. Yun, G. J. Tearney, J. F. de Boer, N. Iftimia, and B. E. Bouma, "High-speed optical frequency-domain imaging," Opt. Express 11(22), 2953-2963 (2003).

30. M. A. Choma, K. Hsu, and J. A. Izatt, "Swept source optical coherence tomography using an all-fiber 1300-nm ring laser source," J. Biomed. Opt. 10(4), 044009 (2005).

31. R. Huber, M. Wojtkowski, K. Taira, J. G. Fujimoto, and K. Hsu, "Amplified, frequency swept lasers for frequency domain reflectometry and OCT imaging: design and scaling principles," Opt. Express 13(9), 35133528 (2005).

\#187097 - \$15.00 USD Received 14 Mar 2013; revised 4 Jun 2013; accepted 6 Jun 2013; published 14 Jun 2013 (C) 2013 OSA 1 July 2013 | Vol. 4, No. 7 | DOI:10.1364/BOE.4.001119| BIOMEDICAL OPTICS EXPRESS 1120 
32. W. Y. Oh, S. H. Yun, G. J. Tearney, and B. E. Bouma, "115 kHz tuning repetition rate ultrahigh-speed wavelength-swept semiconductor laser,” Opt. Lett. 30(23), 3159-3161 (2005).

33. R. Huber, M. Wojtkowski, and J. G. Fujimoto, "Fourier Domain Mode Locking (FDML): A new laser operating regime and applications for optical coherence tomography," Opt. Express 14(8), 3225-3237 (2006).

34. R. Huber, D. C. Adler, and J. G. Fujimoto, "Buffered Fourier domain mode locking: Unidirectional swept laser sources for optical coherence tomography imaging at 370,000 lines/s," Opt. Lett. 31(20), 2975-2977 (2006).

35. W. Wieser, B. R. Biedermann, T. Klein, C. M. Eigenwillig, and R. Huber, "Multi-megahertz OCT: High quality 3D imaging at 20 million A-scans and 4.5 GVoxels per second," Opt. Express 18(14), 14685-14704 (2010).

36. B. Potsaid, B. Baumann, D. Huang, S. Barry, A. E. Cable, J. S. Schuman, J. S. Duker, and J. G. Fujimoto, "Ultrahigh speed 1050nm swept source/Fourier domain OCT retinal and anterior segment imaging at 100,000 to 400,000 axial scans per second," Opt. Express 18(19), 20029-20048 (2010).

37. V. Jayaraman, G. D. Cole, M. Robertson, A. Uddin, and A. Cable, "High-sweep-rate 1310 nm MEMS-VCSEL with $150 \mathrm{~nm}$ continuous tuning range," Electron. Lett. 48(14), 867-868 (2012).

38. I. Grulkowski, J. J. Liu, B. Potsaid, V. Jayaraman, J. Jiang, J. G. Fujimoto, and A. E. Cable, "High-precision, high-accuracy ultralong-range swept-source optical coherence tomography using vertical cavity surface emitting laser light source," Opt. Lett. 38(5), 673-675 (2013).

39. V. Jayaraman, J. Jiang, H. Li, P. J. S. Heim, G. D. Cole, B. Potsaid, J. G. Fujimoto, and A. Cable, "OCT imaging up to $760 \mathrm{kHz}$ axial scan rate using single-mode 1310nm MEMS-tunable VCSELs with 100nm tuning range," in Conference on Lasers and Electro-Optics, Technical Digest (CD) (Optical Society of America, 2011), paper PDPB2.

40. B. R. Biedermann, W. Wieser, C. M. Eigenwillig, T. Klein, and R. Huber, "Dispersion, coherence and noise of Fourier domain mode locked lasers," Opt. Express 17(12), 9947-9961 (2009).

\section{Introduction}

Optical coherence tomography (OCT) performs micrometer-scale, cross-sectional and threedimensional imaging by measuring the echo time delay of backscattered light [1]. Internal body imaging was enabled by the development of fiber-optic based OCT endoscopes [2,3]. In vivo endoscopic OCT imaging is challenging because fast optical scanning must be implemented inside a miniaturized imaging probe. Many scanning mechanisms have been realized in catheter based endoscopic OCT systems, such as proximal rotation of a torque cable and fiber with distal micro-prism [2,4-11], swinging the distal fiber tip by a galvanometric plate [12], swinging the fiber on a cantilever by piezoelectric transducer (PZT) actuators [13-15], and beam scanning using microelectromechanical systems (MEMS) [1619].

Imaging using proximal rotary actuation can cover a large area with a simple scanner configuration and is used in most endoscopic OCT applications, but the scanning may be hindered by bending the catheter because the rotation is transmitted from the proximal motor through a long torque cable to the distal imaging optics. Non-uniform rotation resulting from torsional flexibility and friction throughout the length of the torque cable limits the image quality even if the transverse optical resolution is high. The scanning speed is also limited because the torque cable can vibrate when operated at rotary speeds higher than a few thousand revolutions per minute (rpm). Distal scanning methods using PZT or MEMS based actuators, on the other hand, can provide micron-level precision scanning because the mechanical motion can be directly controlled. However these methods usually have a limited imaging area because the size of the scanner is limited by the catheter size. With advances in micromotor technology, imaging using distal rotary scanning can be achieved, which can provide large scanning area while maintaining high speed, uniform rotation without degrading the image quality. Micromotor catheters for ultrahigh resolution in vivo OCT imaging were demonstrated in 2004 demonstrating imaging at and 2 fps with 1000 axial scans per frame [20,21]. More recently, micromotor catheters and have been used in biomedical applications such as studying smoke induced airway injury [22] and combined fluorescent contrast for intravascular atherosclerotic imaging [23]. Recently, Li, et al. have developed a miniaturized OCT catheter using a two-phase micromotor and demonstrated an imaging speed of $52 \mathrm{fps}$ with 980 axial scans per frame in ex vivo pig bronchus and 208 fps with 208 axial scans per frame in the human finger [24]. This study demonstrated very high frame rates, however the sampling density was limited at higher frame rates due the OCT system imaging speed [24].

\#187097 - \$15.00 USD Received 14 Mar 2013; revised 4 Jun 2013; accepted 6 Jun 2013; published 14 Jun 2013 (C) 2013 OSA 1 July 2013 | Vol. 4, No. 7 | DOI:10.1364/BOE.4.001119| BIOMEDICAL OPTICS EXPRESS 1121 
Wavelength-swept lasers can provide high axial scan / sweep rates while maintaining both narrow linewidth and broad wavelength tuning range and are a key technology for ultrahigh speed swept source OCT [25-32]. Fourier domain mode-locking (FDML), developed by Huber et al., in 2006 was a new technology for wavelength-swept lasers that overcomes fundamental tuning speed limits set by cavity round trip times and enabled high speed OCT imaging [33]. An FDML laser uses a cavity with a long fiber delay line and a fiber FabryPerot tunable filter (FFP-TF) whose sweep rate is synchronized with the roundtrip time of light in the cavity. The long fiber delay line stores the entire frequency sweep in the laser cavity and different sweep frequencies return to the FFP-TF at the time when the filter is tuned to transmit them [33]. Multi-megahertz imaging speed was attained with FDML-based OCT by using multiple buffering stages to increase sweep rate [34,35]. Short cavity swept lasers, with few centimeter cavity lengths, also enable high sweep rate operation [36]. Although the sweep rate of short cavity lasers is limited compared to FDML lasers, short cavity lasers are less sensitive to intracavity dispersion and can usually achieve narrower instantaneous linewidths, which significantly improves the sensitivity roll-off of OCT imaging systems and are especially suitable for long range imaging [36].

Recently, a new swept laser source technology was developed based on MEMS-tunable vertical cavity surface emitting laser (VCSEL) [37]. The VCSEL operates with a single longitudinal mode instead of multiple modes and therefore has an extremely narrow instantaneous linewidth which supports a long imaging range. The micron-scale cavity length of VCSELs and the rapid MEMS response also allows wider-range real time adjustability of both the sweep frequency and wavelength tuning range compared with other lasers. Therefore, the VCSEL is a promising technology for high-speed, long range OCT imaging [38].

In this paper we demonstrate a $3.2 \mathrm{~mm}$ diameter micromotor based miniature catheter for ultrahigh speed endoscopic OCT imaging in an animal model. The micromotor has the advantage of high rotary speed with low driving voltage, ease of rotary speed adjustment and small size. The side-viewing probe can be pulled back over a long distance to acquire threedimensional (3D) data sets covering a large field of view. The VCSEL can provide a $1 \mathrm{MHz}$ axial scan repetition rate which enables a high frame rate while maintaining sufficient axial scans per frame [39]. Using a high speed data acquisition (DAQ) system, ultrahigh speed endoscopic OCT imaging can be achieved and large volume data sets can be acquired with only a few seconds acquisition time.

\section{Methods}

\subsection{Swept source-OCT imaging system}

Figure 1 shows the OCT system used for this experiment. A portion of the laser output was coupled to a Mach-Zehnder interferometer (MZI) to generate interference fringes which are used to calibrate the VCSEL frequency sweep. The MZI was dispersion balanced and set at 1 $\mathrm{mm}$ path difference and fringes were detected by a modified $200 \mathrm{MHz}$ dual-balanced photodetector to generate phase information to recalibrate the OCT interference fringe signals. The detector was modified from a commercially available $350 \mathrm{MHz}$ dual balanced detector (Thorlabs, Inc.) to increase the transimpedance gain by $\sim 2 \mathrm{X}$ to $2.4 \mathrm{kOhms}$ and trading off the bandwidth to $200 \mathrm{MHz}[15,36]$. The OCT system consisted of a dual-balanced Michelson interferometer with a pair of optical circulators and a 50/50 fiber-optic splitter (AC Photonics, Inc.) $[5,15,34]$. The OCT signal was acquired with the same modified photodetector. 


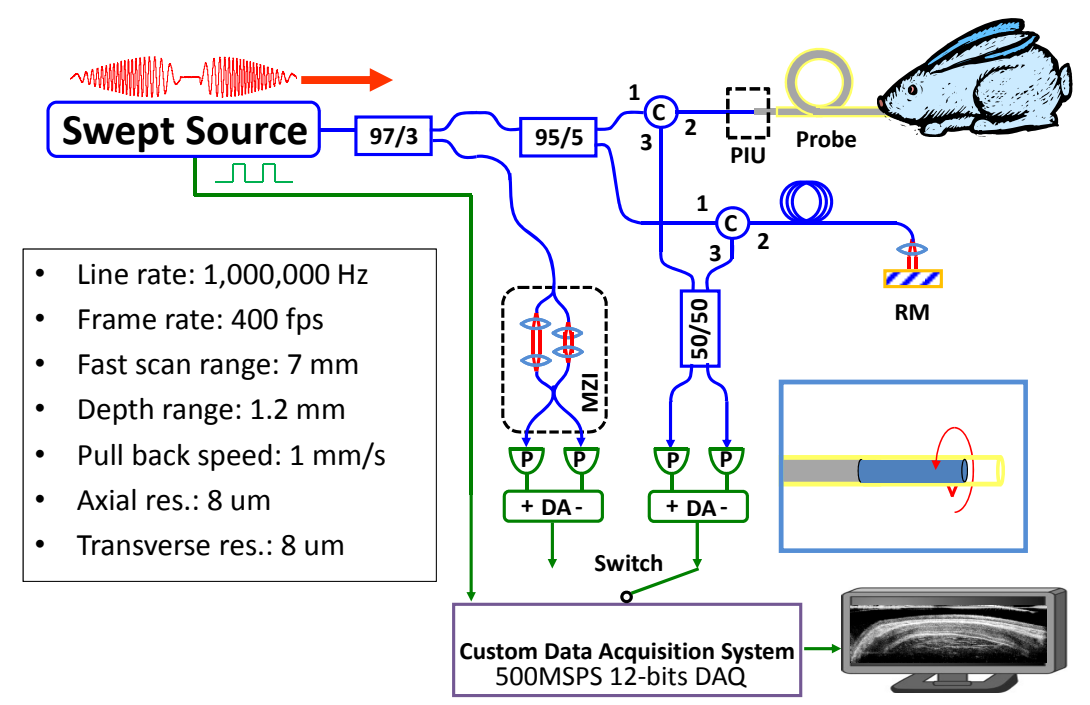

Fig. 1. Schematic of swept source OCT system (optics: blue; electronics: green) and a summary of the system performance (the depth range, axial resolution, and transverse resolution are the numbers in tissue). The inset showing the catheter indicates the rotary scanning direction of the focused spot. C: circulator; MZI: Mach-Zehnder interferometer; RM: reference mirror; DA: differential amplifier; P: photodetector; PIU: patient interface unit.

To enable real time image preview, the MZI calibration interference fringes were acquired once prior to the OCT signal acquisition and the OCT signal was recalibrated by resampling and spline interpolation using the MZI calibration. The MZI calibration and OCT signals were digitized using a high speed 12 bit A/D card with 500 MSPS sample rate (AlazarTech, Inc.). The power on the sample was approximately $20 \mathrm{~mW}$.

\subsection{VCSEL operating at $1 \mathrm{MHz}$ axial scan rate}

Figures 2(a) and 2(b) show a schematic of the VCSEL swept laser (Praevium Research, Inc. and Thorlabs, Inc.) system and device structure. The quantum well active region of the VCSEL was optically pumped at $980 \mathrm{~nm}$ through a wavelength-division multiplexer and wavelength tuning was performed by electrostatic deflection of a MEMS tunable filter. The resonant frequency of the MEMS tunable filter was approximately $500 \mathrm{kHz}$ and it was driven with a sinusoidal waveform at $500 \mathrm{kHz}$. The forward and backward sweeps were both used for image acquisition to achieve an effective sweep rate of $1 \mathrm{MHz}$. The laser output was amplified with two semiconductor optical amplifiers (SOAs, Thorlabs, Inc.) before the OCT system. Two SOAs were used because of wavelength mismatches and limited gain in available single SOAs. The amplified spontaneous emission (ASE) spectrum of the first booster SOA had a peak wavelength at 1,270 nm and a full width at half maximum (FWHM) of $60 \mathrm{~nm}$. The first booster ASE was used as a pre-amplification stage that increased the power in shorter wavelength range and matched the ASE spectrum of the second booster SOA. The ASE spectrum of the second SOA had a peak wavelength at $1,310 \mathrm{~nm}$ and a FWHM of $90 \mathrm{~nm}$. The second booster SOA had higher gain and was used to amplify the output from the first booster SOA. With the wavelengths matched, the VCSEL output can be amplified without decreasing the overall FWHM bandwidth. A redesign of the SOA to improve wavelength matching and gain should allow a single SOA to be used in the future. By tuning the driving current of the first and second SOAs, the FWHM of the output spectrum and the output power could be adjusted based on the application requirements. The maximum output power was $110 \mathrm{~mW}$ if both SOAs were driven at their maximum driving current. However, in this study, the SOAs were set to optimize sweep range and the average output 
power after the booster stage was $40 \mathrm{~mW}$. Figure 2(c) shows the time integrated VCEL output spectrum measured by an optical spectrum analyzer. The central wavelength was $\sim 1310 \mathrm{~nm}$ and the total sweep range was $107 \mathrm{~nm}$, with a $70 \mathrm{~nm}$ FWHM. Figure 2(d) shows the fringe signal from a Mach-Zehnder interferometer. The duty cycle was $>90 \%$ with a $1 \mu$ s sweep duration and symmetric forward and backward sweeps.

(a)

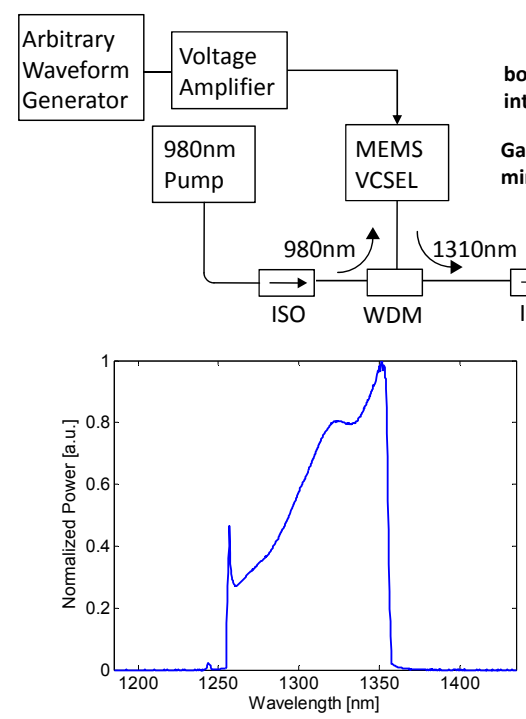

(c)
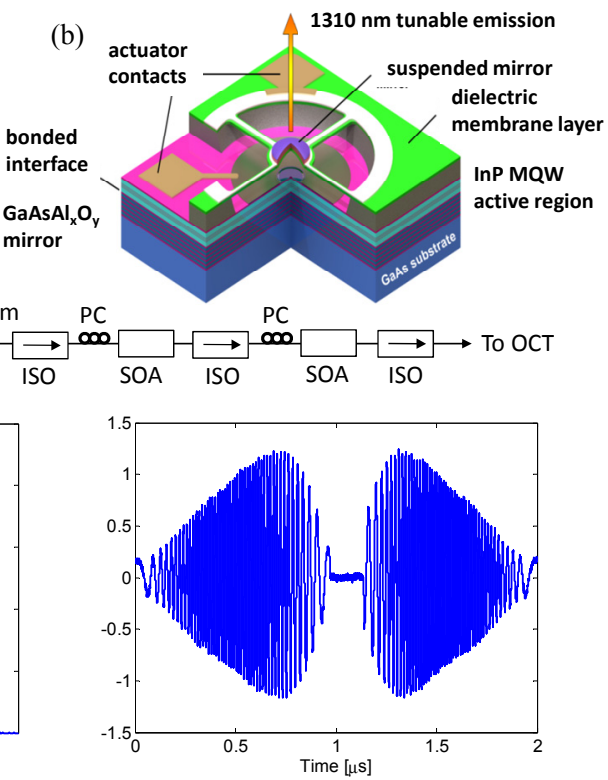

(d)

Fig. 2. (a) Schematic of the VCSEL swept source. (b) The VCSEL device structure. (c) Optical spectrum of the laser output. (d) Interferometric trace from the Mach-Zehnder interferometer. ISO: optical isolator; WDM: wavelength-division multiplexer; SOA: semiconductor optical amplifier; PC: polarization controller.

\subsection{Micromotor-based imaging catheter}

Figure 3(a) shows a schematic of the micromotor-based catheter. A micro-prism was mounted on a $2 \mathrm{~mm}$ diameter, $6 \mathrm{~mm}$ long micromotor (Namiki Precision of California, Inc.). The OCT beam was focused by a fiber-GRIN lens assembly, reflected by the rotating micro-prism $(0.7$ $\mathrm{mm} \times 0.7 \mathrm{~mm}$ ) and focused outside of the catheter sheath. The fiber was mounted in a $3.8 \mathrm{~mm}$ long ferrule and polished at an 8degree angle. There was an air gap before the GRIN lens. The motor and GRIN lens were mounted inside a metal hypotube with a $2.1 \mathrm{~mm}$ inner diameter and $2.37 \mathrm{~mm}$ outer diameter. The hypotube was used to fix the micromotor to a $2.2 \mathrm{~mm}$ outer diameter torque coil (Asahi Intecc, Inc.). The hypotube enables imaging over approximately $70 \%$ of the micro-prism rotation. The FEP plastic sheath which covered the motor and hypotube was $2.8 \mathrm{~mm}$ inner diameter and $3.2 \mathrm{~mm}$ outer diameter (Zeus, Inc.). In order to keep the spot size as small as possible with relatively long working distance from the sheath surface (500 $\mu \mathrm{m}$ in tissue), the GRIN lens (NSG, Inc.) surfaces were polished at 8 degree and 5 degrees and the pitch of the lens was reduced to 0.15 . The spot size was $8 \mu \mathrm{m}$ (FWHM) in tissue.

The micromotor was a three-phase and brushless motor design with a terminal resistance of $\sim 100 \Omega$, which allowed high rotation uniformity and much longer lifetime compared to brushed motor. The maximum no-load speed and the stall torque of the micromotor were $82,000 \mathrm{rpm}$ and $0.0033 \mathrm{mN} \cdot \mathrm{m}$ respectively, indicating the micromotor could provide a torque of $4 \times 10^{-4} \mathrm{mN} \cdot \mathrm{m}$ while operating at $72,000 \mathrm{rpm}$. The speed of the brushless motor is 
limited by the torque the motor can generate and the torque is inversely proportional to the rotary speed of the motor.

By pulling the optical and motor assembly from the proximal end of the torque coil during the rotary image acquisition, a spiral scanning pattern could be performed. The catheter had a $3.2 \mathrm{~mm}$ outer diameter and $18.2 \mathrm{~mm}$ rigid length and could pass through a $3.7 \mathrm{~mm}$ endoscope working channel. The micromotor could rotate uniformly with a driving voltage less than $5 \mathrm{~V}$ at a speed from 1,200 rpm to 72,000 rpm, corresponding to an imaging frame rate from $20 \mathrm{fps}$ to $1,200 \mathrm{fps}$. In this study, a frame rate of $24,000 \mathrm{rpm}$ (400 fps) and a pullback speed of 1 $\mathrm{mm} / \mathrm{s}$ were used to acquire the 3D-OCT data sets. The total length of the torque coil and sheath for the prototype catheter was 2 meters. Figure 3(b) shows a photograph of the assembled imaging catheter. The imaging catheter has an overall transmission rate of $75 \%$ and a parasitic backreflection of $-53 \mathrm{~dB}$, mainly caused by the fiber-air surface in the imaging catheter.

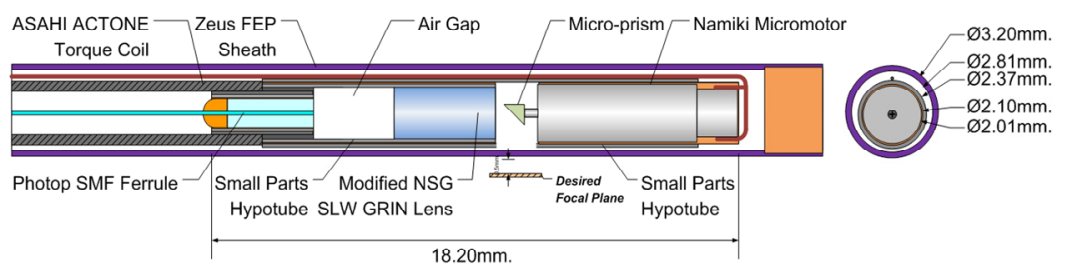

(a)

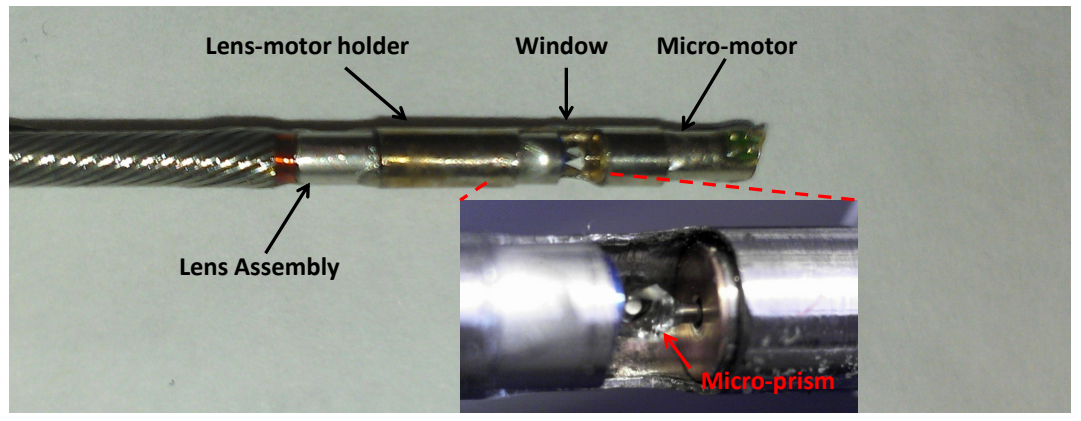

(b)

Fig. 3. (a) Schematic diagram and (b) a photograph of the micromotor probe. The micromotor is held by a hypotube which is open over $70 \%$ of its circumference. The wire is folded back along the hypotube and it is covered with a transparent sheath when fully assembled.

\subsection{High speed and large volume data acquisition}

A 64-bit computer with 32 GB memory was used to support continuous acquisition and streaming of the swept source OCT data. The high speed A/D card (AlazarTech, Inc.) was used to sample the OCT signal at up to 500 MSPS with 12 bit resolution. A customized user interface and data acquisition software were developed in $\mathrm{C}++$ to coordinate instrument control and enable user interaction. The imaging system could acquire OCT data for over 10 seconds at $1 \mathrm{MHz}$ axial scan rate acquiring a data set size larger than $10 \mathrm{~GB}$.

\subsection{Signal processing of the OCT images}

An MZI calibration trace was acquired in the beginning of each imaging session and used to calibrate all of the OCT fringe data for that session. Calibration traces were not required for each axial scan sweep if the MEMS VCSEL was swept at a repetition rate near its resonance frequency. Each MZI sweep contains 1000 A/D sample points, covering both forward and backward scans for each laser sweep. The MZI fringe data was first interpolated by fast Fourier transforming (FFT), zero-padding to 2,048 points, and then inverse Fourier 
transforming (IFFT). The interpolated MZI traces were then Hilbert transformed to extract the phase of the frequency sweep. The phase information was then used to resample the OCT interference signals from equal time intervals to linear phase, or equal frequency interval samples. The OCT interference signals were interpolated to 2,048 points/sweep using FFT/zero-padding/IFFT, then re-sampled using cubic-spline interpolation to be equally sampled in k or frequency using the phase calibration information from the MZI traces. The re-sampled OCT fringe data was then Fourier transformed (FFT) to obtain the axial scans. The axial scans consisted of $\sim 250$ samples, spaced by $\sim 4.8 \mu \mathrm{m}$ with a maximum imaging range of $1.2 \mathrm{~mm}$ in tissue. Images were generated by computing the log of the magnitude of the axial scans.

\section{Results}

\subsection{System performance}

To characterize the system sensitivity, a calibrated $-52 \mathrm{~dB}$ reflection was used in the sample arm. The reference arm power was set to 150-200 microwatts. The sensitivity was measured as the ratio of the peaks of the PSFs to the standard deviation of the noise floor, which was measured with the sample arm blocked. The estimated system losses were $\sim 4 \mathrm{~dB}$ arising from losses in the optics, mirror reflectivity and backcoupling. The measured sensitivity values were not adjusted for these losses. Figure 4 shows the sensitivity roll off and the point spread function from a fixed reflection. The measured sensitivity of the system was $103.1 \mathrm{~dB}$ with an incident power of $20 \mathrm{~mW}$ and the image depth range was $1.65 \mathrm{~mm}$ in air (1.2 $\mathrm{mm}$ in tissue). Figures 4(a) and 4(b) show the sensitivity roll off measured by the 500 MSPS A/D card versus a $1 \mathrm{GHz}$ bandwidth oscilloscope (Tektronix, Inc.). Since the A/D bandwidth was limited, the oscilloscope measurement was used to characterize the VCSEL and detector performance. The sensitivity rolls off $\sim 7 \mathrm{~dB}$ at $1.5 \mathrm{~mm}$ in Fig. 4(a) and was limited by the bandwidth of the $\mathrm{A} / \mathrm{D}$ card $(250 \mathrm{MHz})$. In contrast, the sensitivity was relatively constant over the entire imaging range in Fig. 4(b) when measured using the $1 \mathrm{GHz}$ bandwidth oscilloscope and the R-number was $4 \mathrm{~mm} / \mathrm{dB}$, calculated from the inverse decay constant of the exponential decay curve fitted to the signal maxima of the linear PSFs [40]. The axial resolution was $11 \mu \mathrm{m}$ in air $(8 \mu \mathrm{m}$ in tissue).

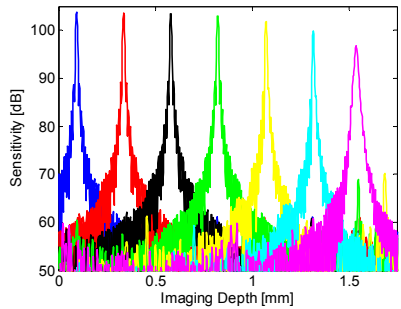

(a)

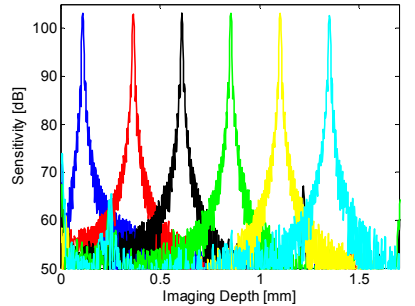

(b)

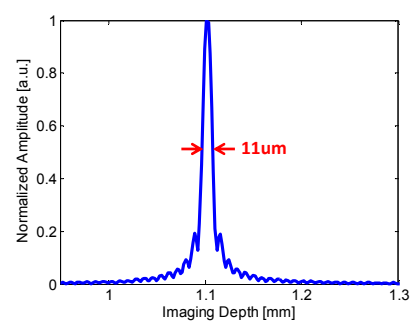

(c)

Fig. 4. (a) Sensitivity roll-off measured using the 500 MSPS A/D card, (b) sensitivity roll-off measured using the $1 \mathrm{GHz}$ oscilloscope, and (c) point spread function of the ultrahigh speed endoscopic OCT system. The point spread function was measured at imaging depth of $\sim 1.1$ $\mathrm{mm}$ and has $11 \mu \mathrm{m}$ axial resolution, corresponding to $8 \mu \mathrm{m}$ in tissue.

\subsection{In vivo rabbit gastrointestinal tract imaging}

To demonstrate the ability to image microscopic structures in the gastrointestinal tract, volumetric 3D-OCT data sets of the esophagus and colon of a female New Zealand White rabbit were acquired in vivo. The animal was anesthetized prior to imaging. Studies were performed under a protocol approved by the Committee on Animal Care (CAC) at M.I.T.

Figure 5 shows examples of cross-sectional images of rabbit esophagus along the rotary direction transverse to the probe, with and without averaging. The micromotor imaging 
catheter could provide extremely high rotary stability and thus enabled contrast enhancement by averaging consecutive images. The image data was displayed in Cartesian coordinates, although the images were acquired by angle scanning the beam and therefore should be in polar form. This display was used because it avoids having a large central empty space at the catheter position, but it produces a transverse distortion of the image with increasing axial distance or depth but enables more efficient visualization than polar images. The axial dimension was divided by the tissue index of refraction $(n=1.38)$ so the axial scale corresponded to physical thickness. The imaging catheter sheath was visible at the top of the images. The sheath outer diameter was $3.2 \mathrm{~mm}$, corresponding to a $\sim 10 \mathrm{~mm}$ circumference, determining the transverse scale at the top of the image. Since only $70 \%$ of the rotary scan produced an image, the transverse direction was cropped to $7.5 \mathrm{~mm}$. Increased axial distance from the sheath corresponded to an increased scan circumference. At an axial distance of 1 $\mathrm{mm}$ from the sheath, the scan circumference is $16.2 \mathrm{~mm}$. This produces an artifact, where features are compressed in the transverse direction with increasing depth. It is also important to note that if the esophagus was not in contact with the sheath around the entire scan, the OCT beam did not intersect the esophagus perpendicularly. The esophageal layers appeared thicker away from the point of contact because the axial scan was at an angle to the layers.
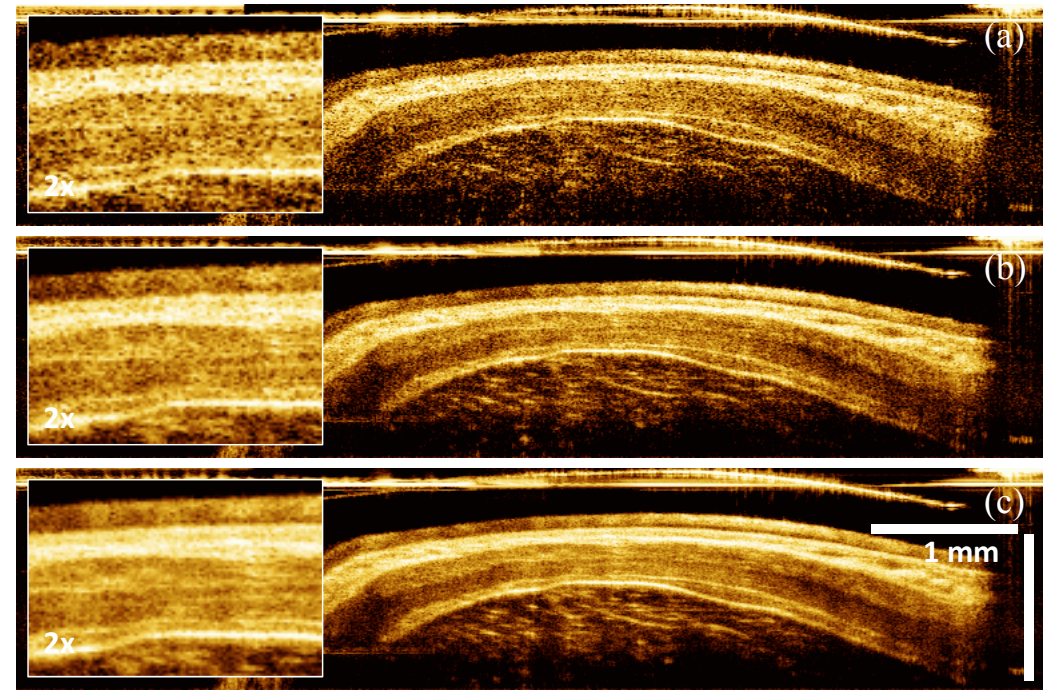

Fig. 5. Image contrast enhancement by averaging. (a) Single image with $2 x$ enlargement in box on left. (b) Averaged of 2 consecutive images, corresponding to a $5 \mu \mathrm{m}$ pullback distance. (c) Averaged of 4 consecutive images, corresponding to a $10 \mu \mathrm{m}$ pullback distance.

Figure 6 shows a 3D-OCT data set from the rabbit esophagus. The ultrahigh speed imaging system enabled the acquisition of very large data sets which cover large areas of tissue with dense spatial sampling. In the data set, 3,000 frames of 2,500 axial scans each were acquired in 7.5 seconds, covering a volume size of $7.5 \mathrm{~mm} \times 7.5 \mathrm{~mm} \times 1.2 \mathrm{~mm}$ (rotary x pullback x axial directions). The pixel spacing was $4 \mu \mathrm{m} \times 2.5 \mu \mathrm{m} \times 4.8 \mu \mathrm{m}$ in the rotary $\mathrm{x}$ pullback $x$ axial directions, respectively. The cross-sectional OCT images (Figs. 6(b)-6(d)) allowed visualization of the entire normal esophageal layers including the epithelium (EP), lamina propria/muscularis mucosa $(\mathrm{LP} / \mathrm{MM})$, submucosa $(\mathrm{SM})$, circular muscle $(\mathrm{Ci})$, and longitudinal muscle (LM). The layered structure in the OCT images correlated well with representative histology of the rabbit esophagus (Fig. 6(e)). The volumetric data set could be processed and displayed in three dimensions. All images shown here were displayed by averaging three consecutive images perpendicular to the viewing direction. Figures 6(a) and 6(c) show the en face and cross-sectional view along the pullback direction respectively. The en face view (Fig. 6(a)) averaged over a depth of $15 \mu \mathrm{m}$, showed features such as vessels over 
a large field of view. Vessels had similar structures to dilated glands in the cross-sectional views, but could be distinguished in the en face images. Periodic motion due to the cardiac cycle was visible in the en face view.
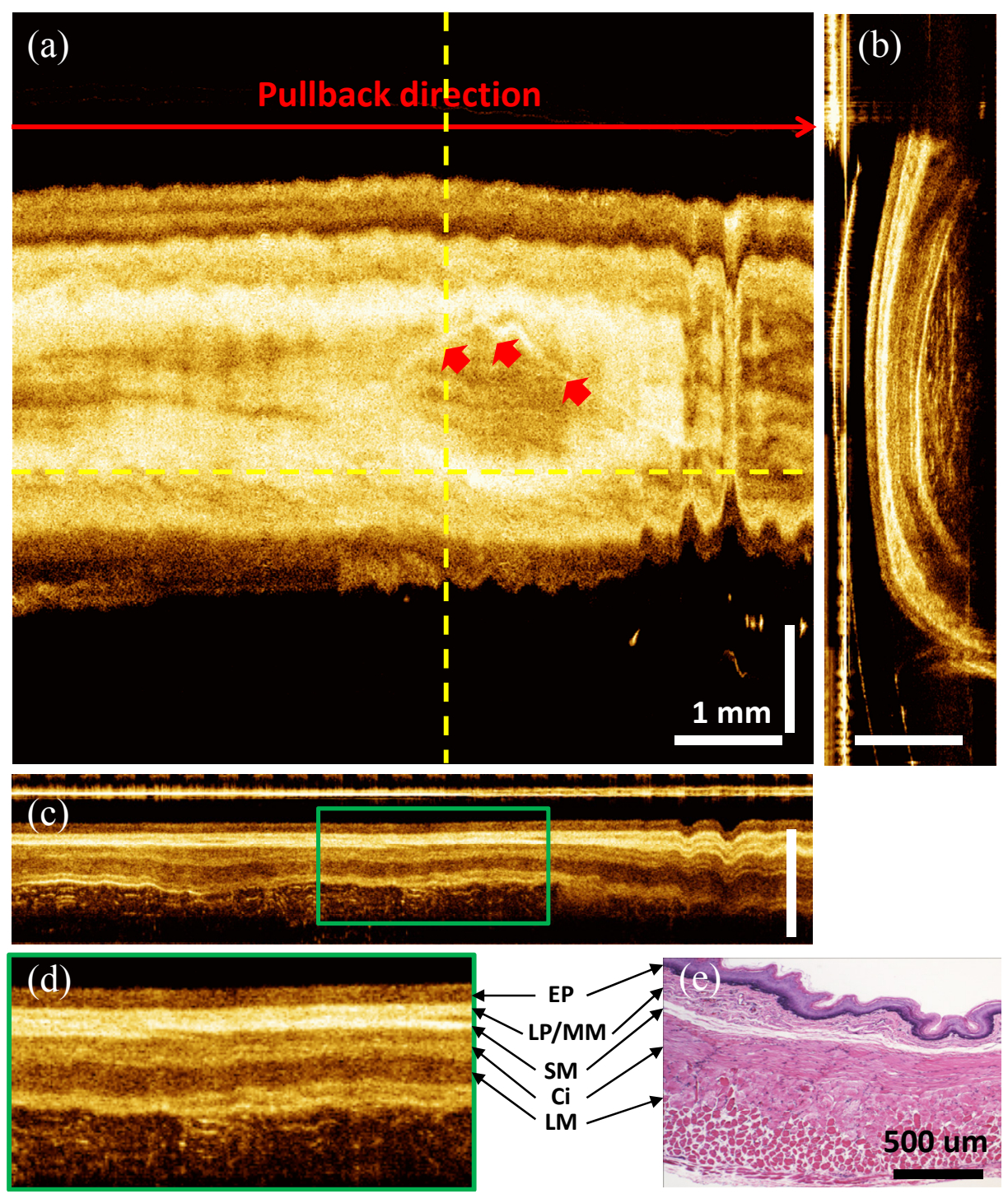

Fig. 6. In vivo volumetric OCT images from rabbit esophagus. (a) En face image averaged over $15 \mu \mathrm{m}$ at depth of $190 \mu \mathrm{m}$. (b) A cross-sectional image transverse to the probe direction averaged over $12 \mu \mathrm{m}$. (c) Longitudinal cross-sectional image along the pullback direction averaged over $7.5 \mu \mathrm{m}$. (d) Enlarged view of (c). (e) Representative histology of the rabbit esophagus. Red arrows: blood vessels. Media 1 shows a 3D rendering and cross-sectional flythrough of volumetric OCT data at the gastro-esophageal junction (GEJ).

The longitudinal cross-sectional images were averaged over $7.5 \mu \mathrm{m}$ (Fig. 6(c)) and provided structural information over a long region of the esophagus, with enhanced imaging contrast due to dense sampling along the pull-back direction. The longitudinal image (Fig. 6(c)) did not have image distortion artifacts which occurred in transverse images (Figs. 5(a)5(c) and Fig. 6(a)) because they were generated by axial scans orientated in a radial plane through the probe. Figure 6(d) shows the enlarged view of the region marked with green box in Fig. 6(c). High speed imaging allowed the visualization of tissue dynamics in vivo. Media 1 
shows a 3D rendering and cross-sectional flythrough (single images without averaging) of a volumetric data set taken at the gastro-esophageal junction (GEJ). Gastric contraction could be observed as motion during the data acquisition.

Figure 7 shows a 3D-OCT data set covering a volume of $7.5 \mathrm{~mm} \times 7.5 \mathrm{~mm} \times 1.2 \mathrm{~mm}$ (rotary $\mathrm{x}$ pullback $\mathrm{x}$ axial directions) in the rabbit colon. All images displayed here were generated by averaging three consecutive images perpendicular to the viewing direction. The en face view at a depth of $300 \mu \mathrm{m}$ (Fig. 7(a)) shows crypt structures in the colon as well as vessels underneath the colon surface. Compared to human tissue, crypts in the rabbit colon are smaller $(\sim 50 \mu \mathrm{m})$ and more tightly packed. Crypts in the rabbit colonic mucosa $(\mathrm{CM})$ were often separated by only a few micrometers of lamina propria, making it usually difficult to identify single crypts in the en face images. Nevertheless, the crypts were visible in some enlarged en face views as shown in the inset of Fig. 7(a). Ultrahigh speed imaging also made 3D-OCT acquisition less sensitive to motion. Figure 7(c) shows a longitudinal cross-sectional image along the pullback direction. Motion artifacts were relatively small throughout the pullback procedure. Therefore, requirements for image post processing, such as frame alignment could be reduced. Media 2 shows the en face flythrough from the surface of the colon to a deeper region.
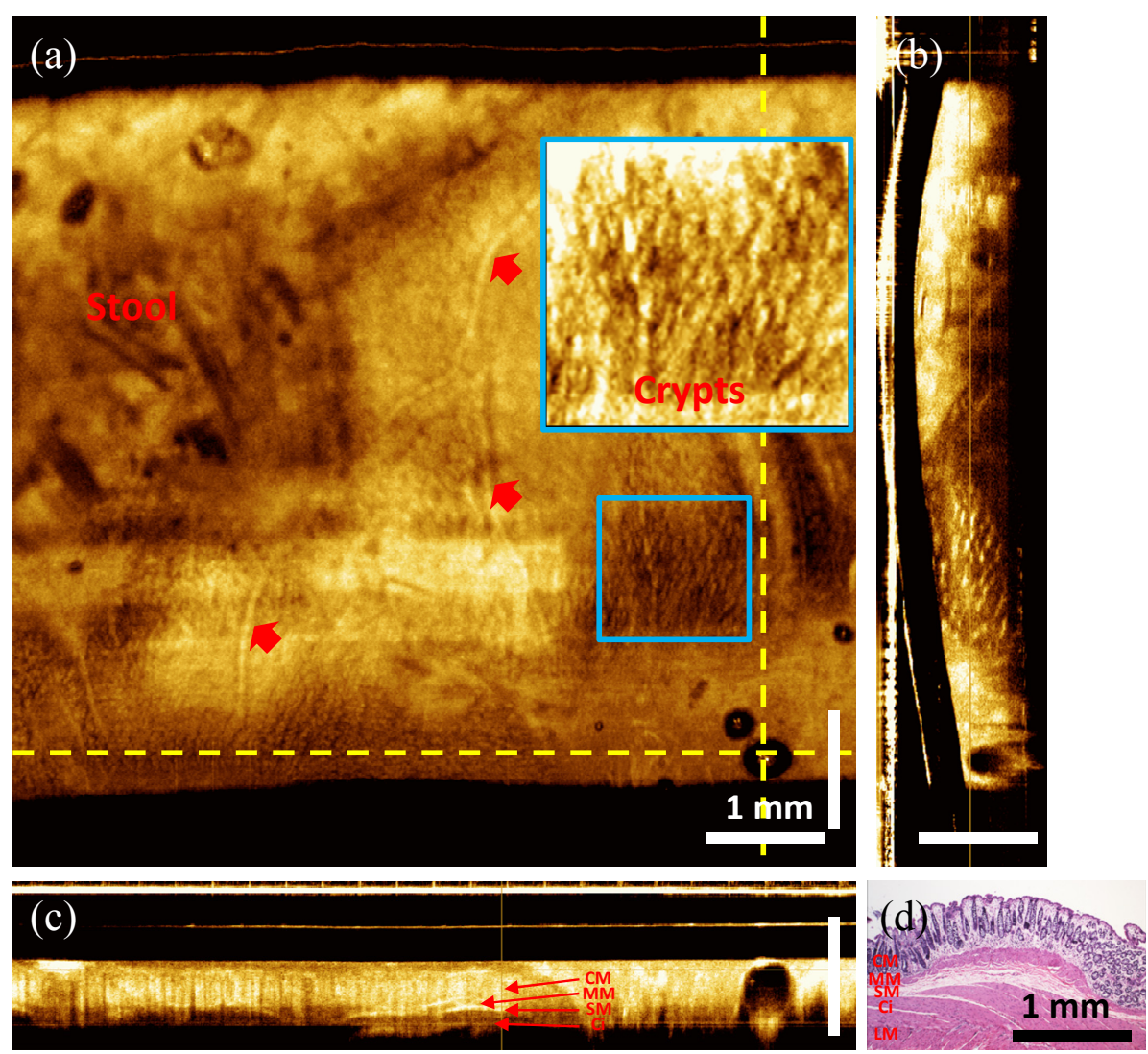

Fig. 7. In vivo volumetric OCT images from rabbit colon. (a) En face image at imaging depth of $300 \mu \mathrm{m}$. (b) Cross-sectional images along the rotary direction. (c) Cross-sectional image along the pullback direction. (d) Representative cross-sectional histology of the rabbit colon with hematoxylin and eosin stain. Red arrows: blood vessels (Media 2. 


\subsection{Ex vivo human colon imaging}

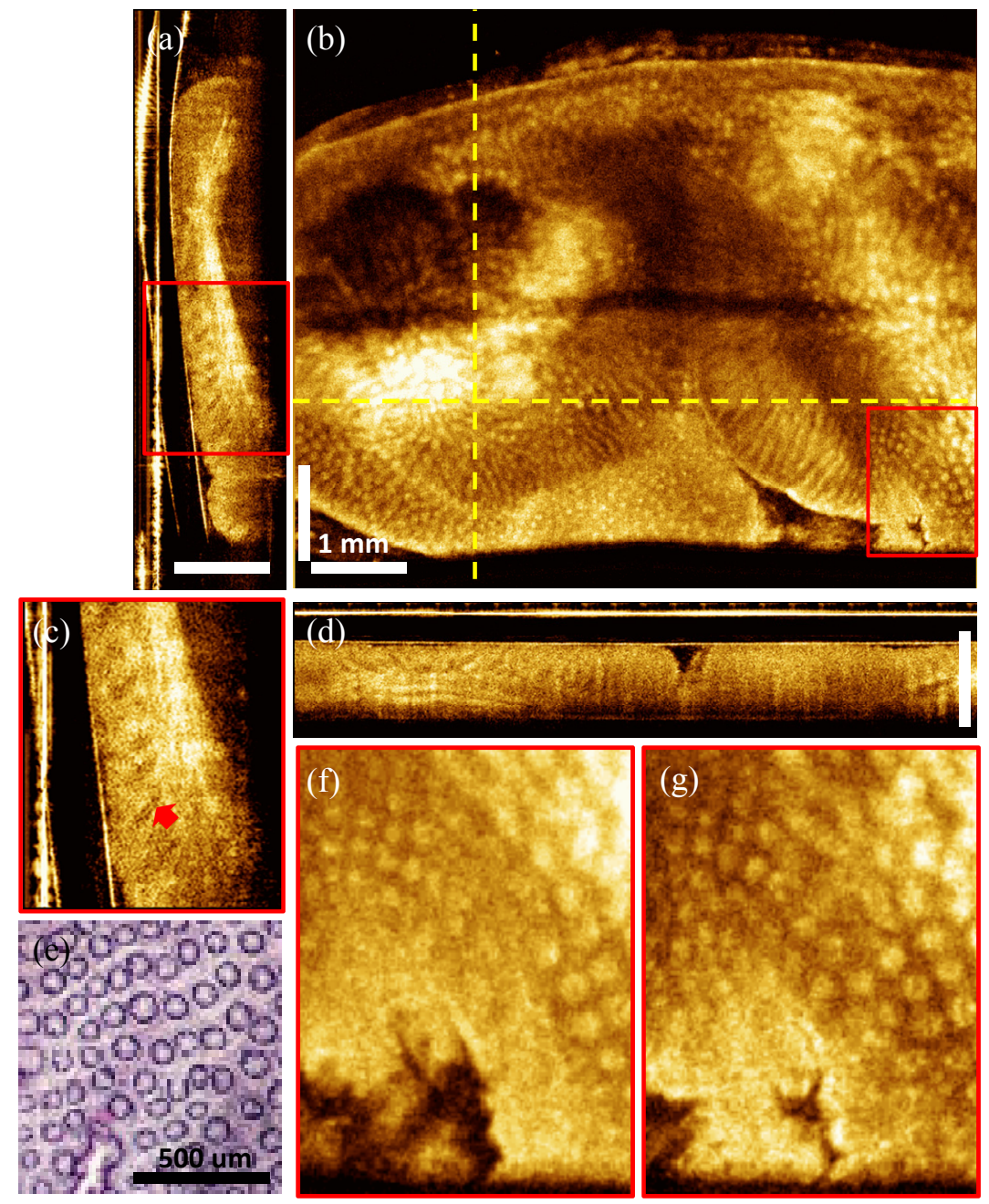

Fig. 8. Ex vivo volumetric OCT images from human colon. (a) Cross-sectional image along the rotary direction. (b) En face image at imaging depth of $350 \mu \mathrm{m}$. (c) Enlarged view of the red box in (a). (d) Cross-sectional image along the pullback direction. (e) Representative en face histology of the human colon with hematoxylin and eosin stain. (f, g) Enlarged view of the en face image at the same location as the red box in (b) at imaging depths of $250 \mu \mathrm{m}$ and $350 \mu \mathrm{m}$. Red arrows: crypts Media 3 .

To evaluate the imaging system for future clinical endoscopic OCT studies, we also imaged human colon specimens ex vivo using the ultrahigh speed OCT system. Specimens which were discarded and not required for clinical diagnosis were obtained under a protocol approved by the Committee for the Use of Humans as Experimental Subjects (COUHES) at M.I.T. and the Investigational Review Board at the Beth Israel Deaconess Medical Center. Fresh unfixed colon specimens were stored in refrigerated DMEM for less than three hours prior to imaging. Figure 8 shows a 3D-OCT data set from freshly excised human colon tissue covering a volume size of $7 \mathrm{~mm} \times 7.5 \mathrm{~mm} \times 1.2 \mathrm{~mm}$ (rotary x pullback x axial direction). Figure 8(b) shows an en face OCT image at a depth of $350 \mu \mathrm{m}$ averaged over 2 pixels, corresponding to a $10 \mu \mathrm{m}$ depth. The individual crypts in the human colon are larger than those in the rabbit colon and were more easily visualized. Figures 8(a) and 8(d) show crosssectional images transverse to the probe along the rotary direction and longitudinal to the 
probe in the pullback direction, respectively. Both the en face and the cross-sectional images show the columnar epithelial structure of the colon (as shown in the enlarged views in Figs. $8(\mathrm{c}), 8(\mathrm{f})$ and $8(\mathrm{~g}))$ and the en face view correlates well with representative histology of human colon shown in Fig. 8(e). Densely sampled volumetric 3D-OCT data sets contained comprehensive information about tissue microstructure. Figure $8(\mathrm{c})$ shows the detailed structure in the crypts. The arrow indicates a narrow line in the crypts, which is possibly the boundary of the crypt lumen. Media 3 shows the en face flythrough of the data set with averaging over 2 pixels, corresponding to a $10 \mu \mathrm{m}$ depth range.

\section{Discussion}

The micromotor based imaging catheter enables high speed scanning with low driving voltage. The distal scanning mechanism is less sensitive to catheter bending, and is therefore more stable than catheters which use proximal rotary actuation. Polarization artifacts are less than in catheters which have proximal rotation because the optical fiber does not twist during actuation. However if the polarization state is not circular, it will still rotate when incident on the tissue because the polarization is reflected from a 90 degree rotating mirror. Therefore if the tissue has polarization dependent backscattering, this may produce artifacts in the images. The current micromotor catheter has the limitation that the wiring and mounting of the micromotor blocks a portion of the imaging field, which requires adjusting the orientation of the imaging probe before image acquisition. The VCSEL light source has both very high sweep rate and broad wavelength tuning range, providing high axial line rate for in vivo imaging and good axial resolution. With the high speed data acquisition, the imaging system can support good imaging depth range with ultrafast line rate. In this study, the effective laser scan repetition rate is $1 \mathrm{MHz}$ and the rotary speed of the micromotor is $400 \mathrm{~Hz}(24,000 \mathrm{rpm})$, so each frame contains 2,500 lines over the circumferential scanning range of $10 \mathrm{~mm}$, corresponding to an axial scan spacing of $\sim 4 \mu \mathrm{m}$ at the surface of the imaging catheter. The pullback speed was $1 \mathrm{~mm} / \mathrm{s}$, corresponding a frame spacing of $2.5 \mu \mathrm{m}$. The data acquisition rate is $500 \mathrm{MS} / \mathrm{s}$ which can provide an imaging depth range of $1.65 \mathrm{~mm}$ in air, or $1.2 \mathrm{~mm}$ in tissue. Imaging range can be improved using high speed detection and data acquisition.

The VCSEL was operated at its resonant frequency, so the sweep-to-sweep repeatability was high enough so the OCT fringe data can be calibrated using a single MZI calibration trace acquired in the beginning of each imaging session. Our previous OCT studies with VCSELs showed noticeable sweep-to-sweep variations in the frequency/wavelength scanning if the MEMS tunable filters in the VCSELs were operated off resonance, so the OCT signal could not be re-calibrated by single MZI interference fringe in this case. Advanced calibration methods such as optical clocking or simultaneous acquisition of MZI and OCT signals are needed to ensure the PSFs not affected by the sweep-to-sweep variations when the VCSEL is not operated at its resonant frequency.

With high frame rate of the imaging system, the acquired data sets are less sensitive to motion, especially when performing in vivo endoscopic imaging. In upper endoscopy, cardiac motion and breathing often induce motion artifacts in OCT images. The high frame rate can reduce the total data acquisition time, while maintaining data acquisition volume. High imaging speed enables rapid acquisition of a densely sampled 3D volumetric data set covering a broad area with minimum motion artifacts. The volumetric data set can be viewed in a variety of orientations. Cross-sectional views provide depth and structural information in the tissue, while en face views can reveal the tissue structure over the field of view at a given depth. Moreover, with the densely sampled data sets, image contrast can be enhanced by image averaging to reduce speckle noise.

The performance of the current prototype can be further improved. The distance from the lens distal surface to the focal plane is relatively long due to the diameter of the micromotor, so the transverse resolution (the spot size on the focal plane) is limited if standard GRIN lenses are used. In this study, a standard GRIN lens with pitch of 0.25 was shortened to 0.15

\#187097 - \$15.00 USD Received 14 Mar 2013; revised 4 Jun 2013; accepted 6 Jun 2013; published 14 Jun 2013 (C) 2013 OSA 1 July 2013 | Vol. 4, No. 7 | DOI:10.1364/BOE.4.001119| BIOMEDICAL OPTICS EXPRESS 1131 
in order to achieve longer effective focal length, which enables a smaller spot size for the long working distance. The transverse resolution can also be improved by using a fiber with higher numerical aperture (NA). A higher NA requires larger diameter optics because the beam from the fiber diverges more rapidly. The micromotor scanning in the rotary direction is highly repeatable, however there are potential discontinuities of motion when the motor and optics are distally pulled back along the longitudinal direction. This can be a limiting factor in the image continuity when using a long catheter because of the friction between the catheter cable and sheath along its length. Typical endoscopic applications in humans would require a 2meter-long catheter. The effects of friction may be reduced by choosing different torque coils or sheath materials. The pullback speed used in this study was $1 \mathrm{~mm} / \mathrm{s}$, which is $1.6 x$ slower than the minimum scanning speed required to achieve Nyquist sampling given the $8 \mu \mathrm{m}$ spot size. The slower pullback speed was used to demonstrate image averaging to reduce speckle and faster pullback speeds can be used in the future. Finally, the rigid length of the distal catheter, including the micromotor and optics is $18.2 \mathrm{~mm}$ and the outer diameter is $\sim 3.2 \mathrm{~mm}$. The imaging catheter can be inserted through an endoscope with a $3.7 \mathrm{~mm}$ diameter working channel, but is still too large to be introduced through the $2.8 \mathrm{~mm}$ working channel of most commonly used esophagogastroduodenal (EGD) endoscopes. The endoscope working channel has a sharp radius bend at the proximal end which requires either a short rigid length or a smaller catheter outer diameter. Therefore, the size of the catheter needs to be reduced to enable use with the more common $2.8 \mathrm{~mm}$ working channels. Alternately, the catheter could be used with a daughter scope, and carried on the side of the standard endoscope.

In conclusion, we demonstrated an ultrahigh speed endoscopic OCT imaging system with record $400 \mathrm{fps}$ frame rate using a micromotor based imaging catheter, a MEMS-tunable VCSEL light source and a high speed data acquisition system. The system can support 400 frames per second with $1 \mathrm{MHz}$ axial line rate, $11 \mu \mathrm{m}$ axial resolution, $7 \mu \mathrm{m}$ transverse resolution and $1.65 \mathrm{~mm}$ imaging depth range in air, corresponding to $8 \mu \mathrm{m}$ axial resolution, 8 $\mu \mathrm{m}$ transverse resolution and $1.2 \mathrm{~mm}$ imaging depth range in tissue. The micromotor can operate 1,200-72,000 rpm (corresponding to 20-1,200 fps) so even faster frame rates can be achieved by trading off pixel density. High imaging speed was demonstrated in vivo in the rabbit esophagus and colon as well as ex vivo in human colon specimens, enabling the visualization of microscopic features. Three dimensional endoscopic OCT data sets enable powerful visualization techniques including speckle reduction by averaging, generation of en face views similar to endoscopic images, and the generation of cross-sectional images with arbitrary orientations. Future improvements in the catheter design and data acquisition technology will allow volumetric imaging with enhanced microscopic resolution and at even higher frame rates and should enable a wide range of clinical 3D-OCT endomicroscopy applications.

\section{Acknowledgments}

We gratefully acknowledge Dr. Robert Marini and Mr. Wayne Au at MIT for assistance with the in vivo rabbit study, Dr. Yuri Sheykin from the Beth Israel Deaconess Medical Center for the preparation of ex vivo human specimens, and Mr. Daniel E. Baird in Knoxville, TN for the discussion of micromotors. Dr. Chao Zhou is currently at Lehigh University. The research was sponsored in part by the National Institutes of Health R01-CA75289-16, R44-CA10106706, R44EY022864-01, R01-EY011289-27, R01-HL095717-04 and R01-NS057476-05; the Air Force Office of Scientific Research FA9550-10-1-0063 and Medical Free Electron Laser Program FA9550-10-1-0551, and German Research Foundation DFG-GSC80-SAOT and DFG-HO-1791/11-1.

\#187097 - \$15.00 USD Received 14 Mar 2013; revised 4 Jun 2013; accepted 6 Jun 2013; published 14 Jun 2013 (C) 2013 OSA 1 July 2013 | Vol. 4, No. 7 | DOI:10.1364/BOE.4.001119| BIOMEDICAL OPTICS EXPRESS 1132 